\title{
On Hardy-Littlewood inequality for Brownian motion on Riemannian manifolds
}

\author{
Alexander Grigor'yan * \\ Department of Mathematics \\ Imperial College \\ London SW7 2BZ \\ United Kingdom \\ email a.grigoryan@ic.ac.uk
}

\author{
Mark Kelbert ${ }^{\dagger}$ \\ European Business Management School \\ University of Wales, Swansea \\ Singleton Park, Swansea SA2 8PP \\ United Kingdom \\ email m.kelbert@swansea.ac.uk
}

March 1998

\section{Introduction}

Let $\left\{X_{i}\right\}_{i \geq 1}$ be a sequence of independent random variables taking the values \pm 1 with the probability $\frac{1}{2}$, and let us set $S_{n}=X_{1}+X_{2}+\ldots+X_{n}$. A classical theorem of Hardy and Littlewood (1914) says that, for any $C>0$ and for all $n$ large enough, we have

$$
S_{n} \leq C \sqrt{n \log n}
$$

with probability 1 . In 1924, Khinchin showed that (1) can be replaced by a sharper inequality

$$
S_{n} \leq \sqrt{(2+\varepsilon) n \log \log n},
$$

for any $\varepsilon>0$. In the view of Khinchin's result, inequality (1) has long been considered as one of a rather historical value. However, the recent results on Brownian motion on Riemannian manifolds give a new insight into it. In this note, we show that an analogue of (1), for the Brownian motion on Riemannian manifolds of the polynomial volume growth, is sharp and, therefore, cannot be replaced by an analogue of (2).

Let $M$ be a smooth connected geodesically complete non-compact Riemannian manifold. Denote by $\Delta$ the Laplace operator of the Riemannian metric of $M$ and by $W_{x}(t)$ the minimal diffusion on $M$ starting at the point $x \in M$ and generated by the operator $\frac{1}{2} \Delta$.

We say that a positive function $R(t)$ on $(0, \infty)$ is an upper radius for $W_{x}(t)$ if, with probability 1 ,

$$
\operatorname{dist}\left(W_{x}(t), x\right) \leq R(t),
$$

for all $t$ large enough, where dist denotes the geodesic distance. For example, if $M=\mathbb{R}^{d}$, then the $d$-dimensional law of the iterated logarithm implies that the function

$$
\sqrt{(2+\varepsilon) t \log \log t}
$$

\footnotetext{
${ }^{*}$ Supported by the EPSRC Advanced Fellowship B/94/AF/1782

${ }^{\dagger}$ Partially supported by the collaborative grant of London Mathematical Society and by the EPSRC Visiting Fellowship
} 
is an upper radius for any $\varepsilon>0$ and is not for $\varepsilon \leq 0$.

Denote by $B(x, R)$ an open geodesic ball on $M$ of radius $R$ with a centre $x \in M$, and by $V(x, R)$ - its Riemannian volume. We say that manifold $M$ has a polynomial volume growth of order $n$ if, for some $x \in M$ and all $r$ large enough,

$$
V(x, r) \leq C r^{n},
$$

for some constant $C$.

The following result was obtained by the authors:

Theorem 1 ([4], [5]) Let $M$ be a geodesically complete non-compact Riemannian manifold. Assume that $M$ has a polynomial volume growth of order $n$. Then, for any $x \in M$, the process $W_{x}(t)$ has the following upper radius:

$$
\sqrt{c_{n} t \log t}
$$

where $c_{n}$ depends only on $n$.

This theorem can be considered as an analogue of the Hardy-Littlewood theorem for the Brownian motion on Riemannian manifolds. It seems remarkable that the polynomial volume growth condition implies, by alone, a restriction on the escape rate of the Brownian motion. Under the more restrictive hypotheses, one can obtain a full analogue of Khinchin's theorem on manifolds. For example, if $M$ has non-negative Ricci curvature, then the function (3) is an upper radius for $\varepsilon>0$ and is not for $\varepsilon<0$ [5, Theorem 1.4].

A natural question arises whether the upper radius (5) of the Hardy-Littlewood type is sharp for some manifold. We prove in this note that, for any $\varepsilon>0$, there exists a manifold of a polynomial volume growth such that the following function

$$
\sqrt{t \log ^{1-\varepsilon} t}
$$

is not an upper radius.

In this example, the manifold is spherically symmetric, which simplifies the proof. However, the situation when the upper radius (5) cannot be essentially improved, seems to be generic in the class of manifolds of a polynomial volume growth.

Added in proof. A similar example for random walks was constructed by M.T. Barlow and A. Perkins 'Symmetric Markov chains in $\mathbb{Z}^{d}$ : how fast can they move?', Probab. Th. Rel. Fields 82 (1989) 95-108.

\section{Lower estimate for upper radius}

Let $p(t, x, y)$ be the heat kernel on a Riemannian manifold $M$, that is, $p(t, x, y)$ is the smallest positive fundamental solution to the heat equation $u_{t}=\Delta u$. Equivalently, $p(t, x, y)$ is a kernel of the semigroup $e^{t \Delta}$ acting in $L^{2}(M)$. In other words, $p(t / 2, x, y)$ is a density of the transition function of the Brownian motion $W_{x}(t)$.

We will assume throughout that $M$ is stochastically complete, that is, for all $t>0$ and $x \in M$,

$$
\int_{M} p(t, x, y) d y=1
$$

Observe that $M$ is stochastically complete provided $M$ is geodesically complete and has a polynomial volume growth (see [2], [3], [7]).

Our example is based on the following theorem which is of interest by itself. 
Theorem 2 Let $M$ be a stochastically complete manifold and let $R(t)$ be an upper radius for $W_{x}(t)$. Then

$$
p(t, x, x) V(x, R(t)) \geq 1-o(1)
$$

as $t \rightarrow \infty$.

Proof. Let us put

$$
\rho_{x}(t)=\operatorname{dist}\left(W_{x}(t), x\right)
$$

Since $R(t)$ is an upper radius, then

$$
\mathbb{P}_{x}\left\{\rho_{x}(t)>R(t)\right\} \rightarrow 0
$$

as $t \rightarrow \infty$. Indeed, the fact that $R(t)$ is an upper radius, implies that, for almost all paths $\omega$ in the path space,

$$
f_{t}(\omega):=\mathbf{1}_{\left\{\rho_{x}(t)>R(t)\right\}} \underset{t \rightarrow \infty}{\longrightarrow} 0 .
$$

Therefore, (8) is implied by the Lebesgue convergence theorem as follows:

$$
\mathbb{P}_{x}\left\{\rho_{x}(t)>R(t)\right\}=\int f_{t}(\omega) d \omega \underset{t \rightarrow \infty}{\longrightarrow} 0 .
$$

Let us observe that

$$
\mathbb{P}_{x}\left\{\rho_{x}(t)>R(t)\right\}=\int_{M \backslash B(x, R(t))} p(t / 2, x, y) d y,
$$

whence

$$
\lim _{t \rightarrow \infty} \int_{M \backslash B(x, R(t))} p(t / 2, x, y) d y=0 .
$$

By using the semigroup identity, the Cauchy-Schwarz inequality, (6) and (9), we obtain (7) as follows:

$$
\begin{aligned}
p(t, x, x) & =\int_{M} p^{2}(t / 2, x, y) d y \\
& \geq \int_{B(x, R(t))} p^{2}(t / 2, x, y) d y \\
& \geq \frac{1}{V(x, R(t))}\left(\int_{B(x, R(t))} p(t / 2, x, y) d y\right)^{2} \\
& =\frac{1}{V(x, R(t))}\left(1-\int_{M \backslash B(x, R(t))} p(t / 2, x, y) d y\right)^{2} \\
& =\frac{1}{V(x, R(t))}(1-o(1)) .
\end{aligned}
$$


Corollary 3 Under the hypotheses of Theorem 2, we have

$$
R(t) \geq V^{-1}\left(x, \frac{1-o(1)}{p(t, x, x)}\right)
$$

as $t \rightarrow \infty$, where $V^{-1}(x, \cdot)$ denotes the inverse function of $V(x, \cdot)$.

Let us show an example of application of (10), for $M=\mathbb{R}^{d}$. In $\mathbb{R}^{d}$, we have $V(r)=c_{d} r^{d}$ and $p(t, x, x)=(4 \pi t)^{-d / 2}$. Thus, (10) yields, for all $t$ large enough,

$$
R(t) \geq \operatorname{const} \sqrt{t} \text {. }
$$

Of course, in view of the law of the iterated logarithm, this result looks not very sharp. However, the estimate (11) is still not empty and even seems to be new. Indeed, the law of the iterated logarithm says that a.s.

$$
\limsup _{t \rightarrow \infty} \frac{\operatorname{dist}\left(W_{x}(t), x\right)}{\sqrt{2 t \log \log t}}=1
$$

which implies that, for any upper radius $R(t)$,

$$
\limsup _{t \rightarrow \infty} \frac{R(t)}{\sqrt{2 t \log \log t}} \geq 1
$$

and

$$
R(t) \geq \sqrt{(2-\varepsilon) t \log \log t}
$$

for a sequence of $t=t_{k} \rightarrow \infty$. On the contrary, estimate (11) holds, although without $\log \log t$, for all $t$ large enough.

Given an increasing function $R(t)$ such that

$$
\frac{R(t)}{\sqrt{t}} \text { is non-decreasing, }
$$

the Kolmogorov-Dvoretzky-Erdös integral test provides the criterion for $R(t)$ to be an upper radius for the Brownian motion in $\mathbb{R}^{d}$ (see [6, Section 4.12]). However, (12) implies already (11). If (12) is not satisfied, then there seems to be no way of deciding whether $R(t)$ is an upper radius or not. Inequality (11) provides a necessary condition for that.

\section{Manifold with 'big' upper radius}

Our main result is the following theorem.

Theorem 4 For any real number $n \geq 2$, there exists a geodesically complete non-compact Riemannian manifold $M$ of bounded curvature and of a polynomial volume growth of order $n$ such that any upper radius $R(t)$ for the Brownian motion on $M$ satisfies the inequality

$$
R(t) \geq \sqrt{c t \log ^{1-\frac{2}{n}} t}
$$

for a sequence of $t=t_{k} \rightarrow \infty$ as $k \rightarrow \infty$ and for some $c>0$. 
By taking $n$ sufficiently large, we obtain, for any $\varepsilon>0$, a manifold on which the function

$$
\sqrt{t \log ^{1-\varepsilon} t}
$$

is not an upper radius.

Proof. We construct a complete Riemannian manifold $M$ with the following properties:

(A) $M$ has a polynomial volume growth of order $n$;

(B) for some $x \in M$, for some sequence $\left\{t_{k}\right\} \rightarrow \infty$ and for all $t=t_{k}$, the following upper bound of the heat kernel holds:

$$
p(t, x, x) \leq \frac{\text { const }}{t^{n / 2}(\log t)^{n / 2-1}} .
$$

(C) $M$ has bounded curvature.

Remark: As was proved in [1, Theorem 6.1], condition (A) implies that, for all $t$ large enough,

$$
p(t, x, x) \geq \frac{\text { const }}{t^{n / 2} \log ^{n / 2} t} .
$$

Comparison with (14) shows that the result of [1, Theorem 6.1] is nearly sharp. As was shown in $[1$, Section 9], one cannot have in general the lower bound

$$
p(t, x, x) \geq \frac{\text { const }}{t^{n / 2}},
$$

assuming only (A). The manifold $M$ of our example is a further elaboration of the example in $[1$, Section 9].

Assuming that we have already such a manifold, Corollary 3, (A) and (B) imply that, for all $t=t_{k}$ as $k \rightarrow \infty$,

$$
\begin{aligned}
R(t) & \geq V^{-1}\left(x, \frac{1-o(1)}{p(t, x, x)}\right) \\
& \geq c^{\prime}\left(\frac{1-o(1)}{p(t, x, x)}\right)^{\frac{1}{n}} \\
& \geq c^{\prime \prime} t^{1 / 2}(\log t)^{\frac{1}{2}-\frac{1}{n}}(1-o(1)),
\end{aligned}
$$

whence (13) follows.

The simplest example of a manifold with a polynomial volume growth of order $n$ is the Euclidean space $\mathbb{R}^{n}$ (provided $n$ is an integer). However, the Euclidean heat kernel on the diagonal is given by

$$
p(t, x, x)=\frac{1}{(4 \pi t)^{n / 2}},
$$

which clearly does not satisfy (14). On the other hand, on the hyperbolic space, the heat kernel decays exponentially as $t \rightarrow \infty$. This suggests the following approach for constructing $M$. The manifold $M$ will be realized as $\mathbb{R}^{d}$, with a special choice of Riemannian metric. This metric is constructed in such a way that makes the curvature negative in appropriate 
places so that the heat kernel becomes smaller than (16), whereas the volume growth is still polynomial.

The dimension $d$ can be anything at least 2. However, in the part of the proof devoted to the computation of the curvature, we assume for simplicity $d=2$.

Let us fix a point $z \in \mathbb{R}^{d}$ and define in $\mathbb{R}^{d}$ the polar coordinates $(r, \varphi)$ centred at $z$. The Riemannian metric of $M$ has the following form:

$$
d s^{2}=d r^{2}+h^{2}(r) d \varphi^{2},
$$

so that $M$ is spherically symmetric. Here $d \varphi$ is the standard metric on $\mathbb{S}^{d-1}$.

The function $h(r)$ is so far any smooth positive function on $(0, \infty)$, such that $h(r)=r$ for $r \leq 1$. This means that the metric in the ball $B(z, 1)$ is exactly Euclidean. We will choose $h(r)$, for $r>1$, in such a way that $M$ has an infinite sequence of long enough shells of constant negative curvature. These shells serve as 'accelerators' for the Brownian motion $W_{z}(t)$ towards infinity and, thus, diminish the probability of $W_{z}(t)$ to return to $z$.

The boundary area of any sphere $\partial B(z, r)$ can be found as

$$
S(r)=\omega_{d} h^{d-1}(r),
$$

where $\omega_{d}$ is the area of the unit sphere in $\mathbb{R}^{d}$. The volume function is obviously given by

$$
V(r)=V(z, r)=\int_{0}^{r} S(\xi) d \xi,
$$

It will be convenient for us to work with the following function:

$$
\sigma(r)=\frac{S(r)}{V(r)}
$$

For $r \leq 1$, we have $\sigma(r)=\frac{d}{r}$. Clearly, $V(r)$ (and, thus, $S(r)$ and $h(r)$ ) can be recovered through $\sigma$, as follows:

$$
V(r)=V(1) \exp \left(\int_{1}^{r} \sigma(\xi) d \xi\right)
$$

Let $\left\{\left(a_{k}, b_{k}\right)\right\}_{k=0}^{\infty}$ be a sequence of intervals on $(0, \infty)$, which do not intersect, go to $\infty$ as $k \rightarrow \infty$ and satisfy the following conditions: for any $k \geq 1$,

$$
1+b_{k-1} \leq a_{k} \leq C b_{k-1},
$$

for a large constant $C$, and

$$
b_{k} \geq e a_{k} .
$$

In fact, the exact recurrence relations between $b_{k-1}, a_{k}$ and $b_{k}$ will be chosen later, in (53) and (46). So far, we assume only (20) and (21). Let us also put for certainty $a_{0}=2$ and $b_{0}=3$.

Assuming that $\left\{a_{k}\right\},\left\{b_{k}\right\}$ are chosen already, let us show that there exists a function $\sigma(r)$ on $(0, \infty)$ satisfying the following conditions:

(i) for $r \leq 1$, we have $\sigma(r)=\frac{d}{r}$;

(ii) $\sigma(r)$ is non-increasing, smooth and positive on $(0, \infty)$;

(iii) on each interval $\left(a_{k}, b_{k}\right)$, the function $\sigma(r)$ is equal to a constant, which will be denoted by $\eta_{k}$; 
(iv) for each $k \geq 1$, the volume function $V(r)$, defined by (19), satisfies the identities

$$
V\left(a_{k}\right)=a_{k}^{n} \quad \text { and } \quad V\left(b_{k}\right)=b_{k}^{n} .
$$

Clearly, for any non-increasing sequence $\left\{\eta_{k}\right\}$ of positive numbers, the function $\sigma(r)$ can be constructed to satisfy (i)-(iii), by a smooth interpolation on $\left(b_{k-1}, a_{k}\right)$ between the constants $\eta_{k-1}$ and $\eta_{k}$. In order to satisfy (iv), it suffices to have (22), for $k=0$, and the following two equations, for all $k \geq 1$ :

$$
\int_{a_{k}}^{b_{k}} \sigma(r) d r=n\left(\log b_{k}-\log a_{k}\right)
$$

and

$$
\int_{b_{k-1}}^{a_{k}} \sigma(r) d r=n\left(\log a_{k}-\log b_{k-1}\right) .
$$

Indeed, as soon as we have (23) and (24), (22) follows, by induction, from (19).

Let us show that (23) and (24) can be, indeed, satisfied. Since $\sigma(r)=\eta_{k}$ on $\left(a_{k}, b_{k}\right)$, then (23) is equivalent to

$$
\eta_{k}=n \frac{\log b_{k}-\log a_{k}}{b_{k}-a_{k}} .
$$

From now on, let us assume that $\eta_{k}$ is defined by (25). Since $\sigma(r)$ varies on $\left(b_{k-1}, a_{k}\right)$ between $\eta_{k}$ and $\eta_{k-1}$, then it is possible to construct $\sigma$, satisfying (24), provided

$$
\eta_{k} \leq n \frac{\log a_{k}-\log b_{k-1}}{a_{k}-b_{k-1}} \leq \eta_{k-1} .
$$

In view of $(25)$, this is equivalent to

$$
\frac{\log b_{k}-\log a_{k}}{b_{k}-a_{k}} \leq \frac{\log a_{k}-\log b_{k-1}}{a_{k}-b_{k-1}} \leq \frac{\log b_{k-1}-\log a_{k-1}}{b_{k-1}-a_{k-1}},
$$

which is true for any increasing sequence of four numbers $a_{k-1}, b_{k-1}, a_{k}, b_{k}$, just by the concavity of log.

Thus, all conditions (i)-(iv) can be satisfied, by a proper choice of function $\sigma(r)$. In the last part of this proof, we will construct $\sigma(r)$ explicitly, in order to control the curvature of $M$. If we forget for a while about the smoothness of $\sigma$, then such a function can be easily written down as follows:

$$
\sigma(r)= \begin{cases}\frac{n}{r}, & b_{k-1}<r<a_{k} \\ \eta_{k}, & a_{k} \leq r \leq b_{k}\end{cases}
$$

(for small $r$, the function $\sigma(r)$ is defined arbitrarily, only satisfying (i) and (ii)). This function is shown on Fig. 1.

In order to verify that function (27) satisfies the conditions (i)-(iv), it suffices to show identity (24) and that function (27) is non-increasing. The former is obvious, whereas the latter amounts to

$$
\eta_{k-1} \geq \frac{n}{r} \geq \eta_{k},
$$

for any $r \in\left(b_{k-1}, a_{k}\right)$, or to

$$
\frac{n}{b_{k}} \leq \eta_{k} \leq \frac{n}{a_{k}} .
$$


In the view of (25), inequality (29) can be rewritten as

$$
\frac{1}{b_{k}} \leq \frac{\log b_{k}-\log a_{k}}{b_{k}-a_{k}} \leq \frac{1}{a_{k}},
$$

which is true again by the concavity of $\log$.

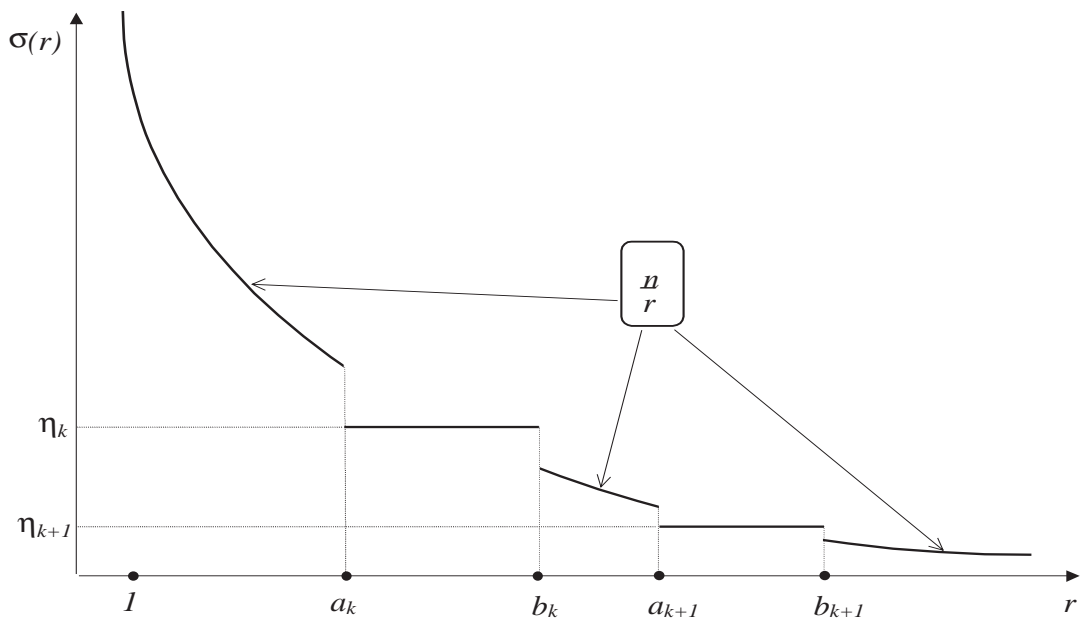

Figure 1 Example of discontinuous $\sigma(r)$

The corresponding function $V(r)$, determined from (19), is as follows:

$$
V(r)= \begin{cases}r^{n}, & b_{k-1}<r<a_{k}, \\ a_{k}^{n} \exp \left(\eta_{k}\left(r-a_{k}\right)\right), & a_{k} \leq r \leq b_{k},\end{cases}
$$

which is sketched in Fig. 2.

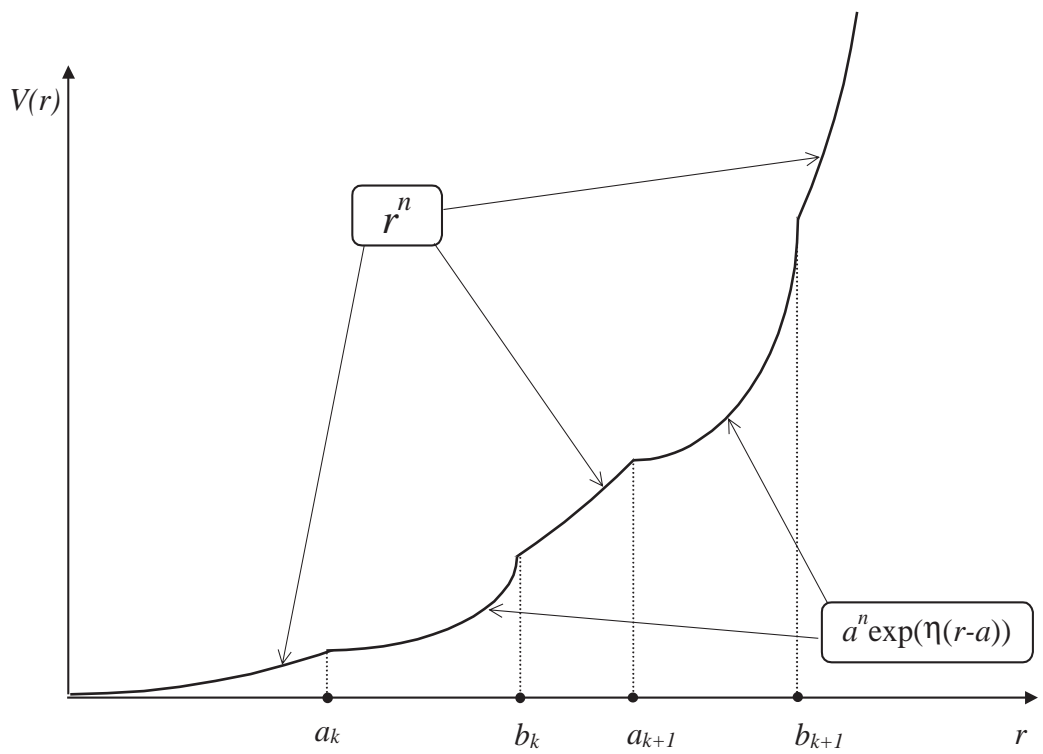

Figure 2 Example of the volume function $V(r)$

Remark: The main role in the construction is played by the intervals $\left(a_{k}, b_{k}\right)$ where $V(r)$ is growing exponentially. If we were to allow discontinuous $\sigma$ then we could put 
$a_{k+1}=b_{k}$ removing thus the layers of the polynomial volume growth. The role of the intervals $\left(b_{k}, a_{k+1}\right)$ is to provide some space for smoothing out the functions $\sigma$ and $V$.

Now we may assume that all conditions (i)-(iv) hold and will show that (A), (B) and (C) can be satisfied, too by further specifying $\left\{a_{k}\right\},\left\{b_{k}\right\}$ and $\sigma(r)$, within the limitations imposed already.

CONDITION (A). Let us prove that, for all $r$ large enough,

$$
V(z, r)=V(r) \leq C r^{n} .
$$

If $r=a_{k}$ or $r=b_{k}$, then it follows from (22). Let us consider the case $r \in\left(a_{k}, b_{k}\right)$. Then, by (19), (iii), (22), (25) and by the concavity of log, we have

$$
\begin{aligned}
V(r) & =V\left(a_{k}\right) \exp \left(\int_{a_{k}}^{r} \sigma(\xi) d \xi\right) \\
& =V\left(a_{k}\right) \exp \left(\eta_{k}\left(r-a_{k}\right)\right) \\
& =a_{k}^{n} \exp \left(n \frac{\log b_{k}-\log a_{k}}{b_{k}-a_{k}}\left(r-a_{k}\right)\right) \\
& \leq a_{k}^{n} \exp \left(n\left(\log r-\log a_{k}\right)\right) \\
& =r^{n} .
\end{aligned}
$$

If $r \in\left(b_{k-1}, a_{k}\right)$, then, by (20), we obtain

$$
V(r) \leq V\left(a_{k}\right)=a_{k}^{n}=\left(\frac{a_{k}}{b_{k-1}}\right)^{n} b_{k-1}^{n} \leq \mathrm{const} r^{n} .
$$

CONDITION (B). To get an upper bound for $p(t, z, z)$, we apply [1, Theorem 8.3], which gives an upper bound of the heat kernel $p(t, z, z)$ on a spherically symmetric manifold $M$, provided the function $\sigma(r)=\frac{S(r)}{V(r)}$ is non-increasing.

Let us define the function $\rho(t)$ from the identity

$$
t=4 \int_{0}^{\rho(t)} \frac{d r}{\sigma(r)} .
$$

Then [1, Theorem 8.3] says that, for any $s \in(0, t)$, the following estimate holds:

$$
p(t, z, z) \leq \frac{2 t}{(t-s) V(\rho(s))} .
$$

Let us define the sequences $\left\{\theta_{k}\right\}$ and $\left\{\tau_{k}\right\}$ as follows (see Fig. 3)

$$
\theta_{k}:=4 \int_{0}^{a_{k}} \frac{d r}{\sigma(r)} \quad \text { and } \quad \tau_{k}:=4 \int_{0}^{b_{k}} \frac{d r}{\sigma(r)}
$$

In other words,

$$
\rho\left(\theta_{k}\right)=a_{k} \quad \text { and } \quad \rho\left(\tau_{k}\right)=b_{k} .
$$

We apply (32) for $t=\tau_{k}$ and for

$$
s=s_{k}:=\tau_{k}-\frac{4}{\eta_{k}^{2}}
$$


This choice of $s$ is motivated by the fact that the ratio

$$
\frac{V\left(\rho\left(s_{k}\right)\right)}{V\left(b_{k}\right)}
$$

is equal to a constant, regardless of $k$ (see below (38)).

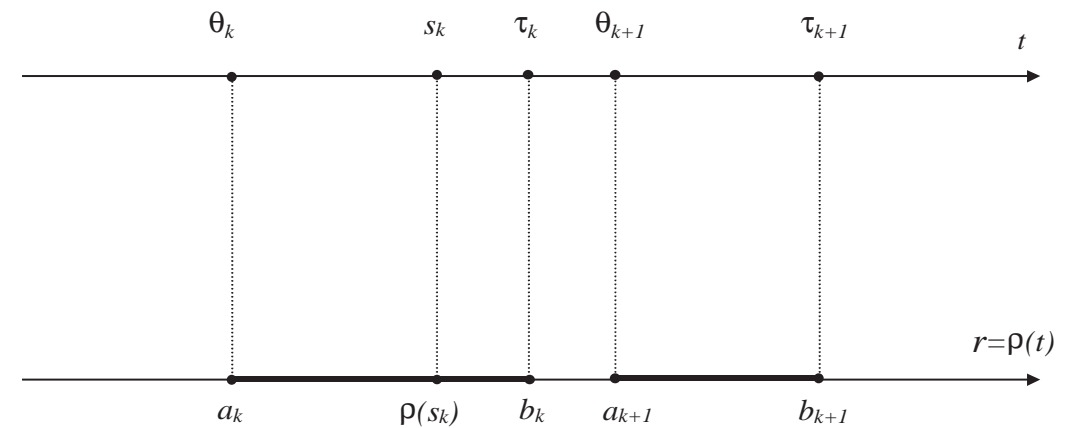

Figure 3 Sequences $\theta_{k}, \tau_{k}$ and $s_{k}$.

Let is first verify that $s_{k} \in\left(\theta_{k}, \tau_{k}\right)$. To that end, it is sufficient to show that

$$
\tau_{k}-\theta_{k} \geq \frac{4}{\eta_{k}^{2}}
$$

We have, by (33) and (iii),

$$
\tau_{k}-\theta_{k}=4 \int_{a_{k}}^{b_{k}} \frac{d r}{\sigma(r)}=4 \frac{b_{k}-a_{k}}{\eta_{k}} .
$$

Thus, by (25) and (21),

$$
\tau_{k}-\theta_{k}=\frac{4}{\eta_{k}}\left(b_{k}-a_{k}\right) \geq \frac{4}{\eta_{k}} \frac{b_{k}-a_{k}}{n \log \frac{b_{k}}{a_{k}}}=\frac{4}{\eta_{k}^{2}},
$$

whence (35) follows.

Since $s_{k} \in\left(\theta_{k}, \tau_{k}\right)$, we have by (31), (33) and (iii)

$$
s_{k}-\theta_{k}=4 \int_{a_{k}}^{\rho\left(s_{k}\right)} \frac{d r}{\sigma(r)}=\frac{4\left(\rho\left(s_{k}\right)-a_{k}\right)}{\eta_{k}},
$$

whence

$$
\rho\left(s_{k}\right)-a_{k}=\frac{\eta_{k}}{4}\left(s_{k}-\theta_{k}\right) .
$$

Let us compute $V\left(\rho\left(s_{k}\right)\right)$, by using successively (22), (30), (37), (34) and (36):

$$
\begin{aligned}
V\left(\rho\left(s_{k}\right)\right) & =a_{k}^{n} \exp \left(\eta_{k}\left(\rho\left(s_{k}\right)-a_{k}\right)\right) \\
& =a_{k}^{n} \exp \left(\frac{\eta_{k}^{2}}{4}\left(s_{k}-\theta_{k}\right)\right) \\
& =a_{k}^{n} \exp \left(\frac{\eta_{k}^{2}}{4}\left(\tau_{k}-\frac{4}{\eta_{k}^{2}}-\theta_{k}\right)\right) \\
& =a_{k}^{n} \exp \left(\frac{\eta_{k}^{2}}{4}\left(4 \frac{b_{k}-a_{k}}{\eta_{k}}-\frac{4}{\eta_{k}^{2}}\right)\right) \\
& =a_{k}^{n} e^{-1} \exp \left(\eta_{k}\left(b_{k}-a_{k}\right)\right) \\
& =e^{-1} V\left(b_{k}\right) \\
& =e^{-1} b_{k}^{n} .
\end{aligned}
$$


Therefore, we have, by (32) and (34),

$$
p\left(\tau_{k}, z, z\right) \leq \frac{2 \tau_{k}}{\left(\tau_{k}-s_{k}\right) V\left(\rho\left(\tau_{k}\right)\right)} \leq \frac{e \eta_{k}^{2} \tau_{k}}{2 b_{k}^{n}}
$$

and

$$
\tau_{k}^{n / 2} p\left(\tau_{k}, z, z\right) \leq \frac{e}{2} \frac{\eta_{k}^{2} \tau_{k}^{n / 2+1}}{b_{k}^{n}}
$$

As follows from (33) and from non-increasing of $\sigma$,

$$
\tau_{k}=4 \int_{0}^{b_{k}} \frac{d r}{\sigma(r)} \leq \frac{4 b_{k}}{\eta_{k}} .
$$

Hence, (39) implies

$$
\tau_{k}^{n / 2} p\left(\tau_{k}, z, z\right) \leq \frac{e}{2} \frac{\eta_{k}^{2}}{b_{k}^{n}}\left(\frac{4 b_{k}}{\eta_{k}}\right)^{n / 2+1}=c\left(b_{k} \eta_{k}\right)^{-n / 2+1} .
$$

Let us observe that, by (25),

$$
b_{k} \eta_{k}=b_{k} \frac{n\left(\log b_{k}-\log a_{k}\right)}{b_{k}-a_{k}} \geq n \log b_{k}-n \log a_{k}
$$

On the other hand, by (40), (25) and (21),

$$
\log \tau_{k} \leq \log \left(\frac{4 b_{k}}{\eta_{k}}\right)=\log \left(4 b_{k} \frac{b_{k}-a_{k}}{n \log \frac{b_{k}}{a_{k}}}\right) \leq \log \left(4 b_{k}^{2}\right)=2 \log b_{k}+\log 4 .
$$

Comparison of (42) and (43) shows that we can have

$$
\log \tau_{k} \leq 2 b_{k} \eta_{k}
$$

provided

$$
b_{k} \geq 2 a_{k}^{n /(n-1)} .
$$

Thus, let us set

$$
b_{k}=e a_{k}^{n /(n-1)},
$$

which satisfies also (21). Inequalities (41) and (44) imply

$$
\tau_{k}^{n / 2} p\left(\tau_{k}, z, z\right) \leq c^{\prime}\left(\log \tau_{k}\right)^{-n / 2+1}
$$

and

$$
p\left(\tau_{k}, z, z\right) \leq \frac{c^{\prime}}{\tau_{k}^{n / 2}\left(\log \tau_{k}\right)^{n / 2-1}}
$$

which was to be proved.

CONDITION (C). We will show that the manifold $M$ has bounded curvature, for a certain choice of $\sigma(r)$. For simplicity, we will assume in this part of the proof that $d=2$. Then the Gauss curvature of $M$ can be easily computed from (17):

$$
K=K(r)=-\frac{h^{\prime \prime}}{h}=-\frac{S^{\prime \prime}}{S}=-\frac{V^{\prime \prime \prime}}{V^{\prime}}=-\left(\frac{\sigma^{\prime \prime}}{\sigma}+3 \sigma^{\prime}+\sigma^{2}\right) .
$$


Since $\sigma(r)=\eta_{k}=$ const for $r \in\left(a_{k}, b_{k}\right)$, then the curvature in the annulus $\left\{a_{k}<r<b_{k}\right\}$ is equal to $-\eta_{k}^{2}$. Since, by (25) and (46),

$$
\eta_{k} \sim \frac{\log b_{k}}{b_{k}} \underset{k \rightarrow \infty}{\longrightarrow} 0,
$$

then the curvature in all annuli $\left\{a_{k}<r<b_{k}\right\}$ remains negative, bounded and, moreover, vanishes at infinity.

To control the curvature in the annuli $\left\{b_{k-1}<r<a_{k}\right\}$, we have to carefully construct $\sigma(r)$ for $r \in\left(b_{k-1}, a_{k}\right)$. (Let us remark the part $b_{k-1}+1 \leq a_{k}$ of condition (20) was imposed from the very beginning to ensure that the length of interval $\left(b_{k-1}, a_{k}\right)$ is bounded away from zero, which helps to make the curvature bounded.) The following restrictions, which are mostly implied by (i)-(iv), should be taken care of:

(a) $\sigma(r)$ is non-increasing and smooth;

(b) the "boundary" conditions:

$$
\sigma\left(b_{k-1}\right)=\eta_{k-1} \quad \text { and } \quad \sigma\left(a_{k}\right)=\eta_{k}
$$

(c) the identity (24):

$$
\int_{b_{k-1}}^{a_{k}} \sigma(r) d r=n\left(\log a_{k}-\log b_{k-1}\right) .
$$

(d) as follows from (47) and from the decreasing of $\sigma$, the boundedness of curvature will be implied by

$$
\sup _{b_{k-1}<r<a_{k}}\left|\frac{\sigma^{\prime \prime}(r)}{\sigma(r)}\right|+\sup _{b_{k-1}<r<a_{k}}\left|\sigma^{\prime}(r)\right|<C,
$$

where the constant $C$ should be the same for all $k$ large enough.

Let us fix a function $\psi(r) \in C^{\infty}(\mathbb{R})$ such that $\psi(r)=1$ for $r \leq 0, \psi(r)=0$ for $r \geq 1$, and $\psi(r)$ is monotonically decreasing on $(0,1)$. Let us denote

$$
I:=\int_{0}^{1} \psi(r) d r .
$$

We define $\sigma(r)$ on $\left(b_{k-1}, a_{k}\right)$ as follows, for $k$ large enough (see Fig. 4)

$$
\sigma(r)=\eta_{k}+\left(\eta_{k-1}-\eta_{k}\right) \psi\left(\frac{1}{\alpha_{k}} \frac{r-b_{k-1}}{a_{k}-b_{k-1}}\right),
$$

where $\alpha_{k} \in(0,1]$ will be chosen below. Clearly, function (52) satisfies (a) and (b). 


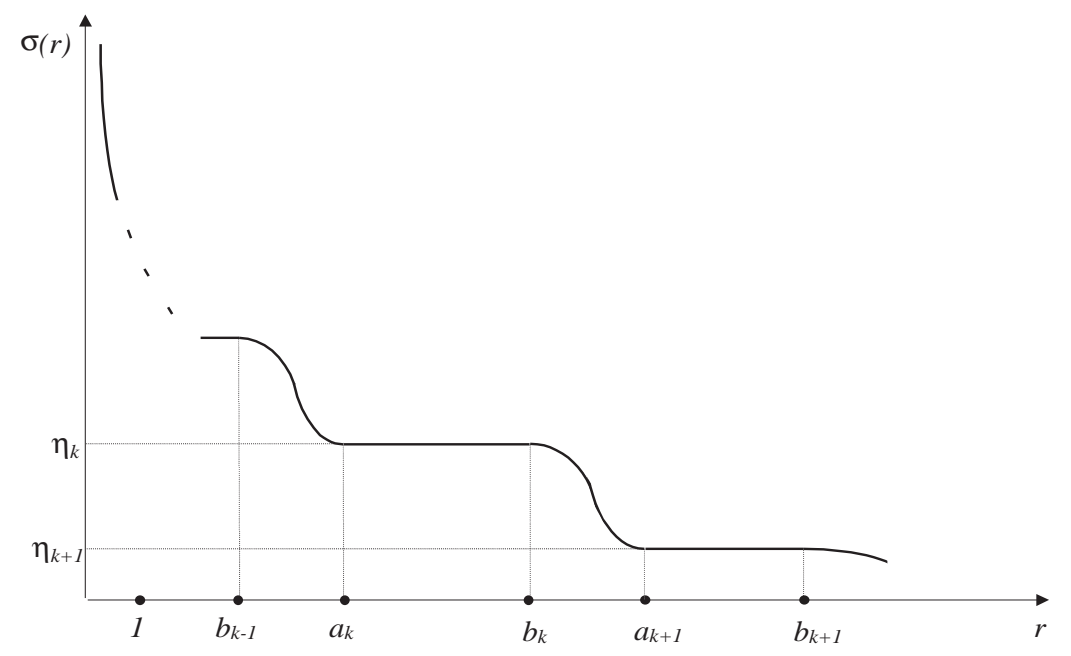

Figure 4 Smooth function $\sigma(r)$

To check (c), let us compute the integral of $\sigma$ :

$$
\int_{b_{k-1}}^{a_{k}} \sigma(r) d r=\left(a_{k}-b_{k-1}\right)\left(\eta_{k}+\left(\eta_{k-1}-\eta_{k}\right) \alpha_{k} I\right) .
$$

From here and (50), we can find $\alpha_{k}$. To simplify the computations, let us set

$$
a_{k}=2 b_{k-1},
$$

which satisfies (20) and implies, by (46),

$$
b_{k}=c b_{k-1}^{n /(n-1)} .
$$

Then (50) acquires the form

$$
\int_{b_{k-1}}^{a_{k}} \sigma(r) d r=n \log 2
$$

and we find

$$
\alpha_{k}=\frac{\frac{n \log 2}{b_{k-1}}-\eta_{k}}{I\left(\eta_{k-1}-\eta_{k}\right)} .
$$

By (26), we have $\alpha_{k}>0$. Let us show that for $k$ large enough, $\alpha_{k} \leq 1$. As is clear from (48) and (54),

$$
\eta_{k} \sim \frac{\log b_{k}}{b_{k}} \sim c^{\prime} \frac{\log b_{k-1}}{b_{k-1}^{n /(n-1)}}, \quad k \rightarrow \infty
$$

Therefore,

$$
\eta_{k}=o\left(\frac{1}{b_{k-1}}\right) \quad \text { and } \quad \eta_{k}=o\left(\eta_{k-1}\right), \quad k \rightarrow \infty,
$$

and we obtain from (55) and (48), that

$$
\alpha_{k} \sim \frac{n}{I} \frac{\frac{\log 2}{b_{k-1}}}{\frac{\log b_{k-1}}{b_{k-1}}}=\frac{c^{\prime \prime}}{\log b_{k-1}}, \quad k \rightarrow \infty .
$$

Thus, $\alpha_{k} \rightarrow 0$ as $k \rightarrow \infty$, and, for $k$ large enough, $\alpha_{k}<1$. 
Now, let us verify (d). For the first derivative of $\sigma$, we have, for $r \in\left(b_{k-1}, a_{k}\right)$,

$$
\sigma^{\prime}(r)=\frac{\eta_{k-1}-\eta_{k}}{\alpha_{k}\left(a_{k}-b_{k-1}\right)} \psi^{\prime}\left(\frac{1}{\alpha_{k}} \frac{r-b_{k-1}}{a_{k}-b_{k-1}}\right) .
$$

Therefore, by (48), (56) and (53),

$$
\left|\sigma^{\prime}(r)\right| \leq C\left(\frac{\log b_{k-1}}{b_{k-1}}\right)^{2} \underset{k \rightarrow \infty}{\longrightarrow} 0 .
$$

In particular, $\left|\sigma^{\prime}\right|$ remains bounded.

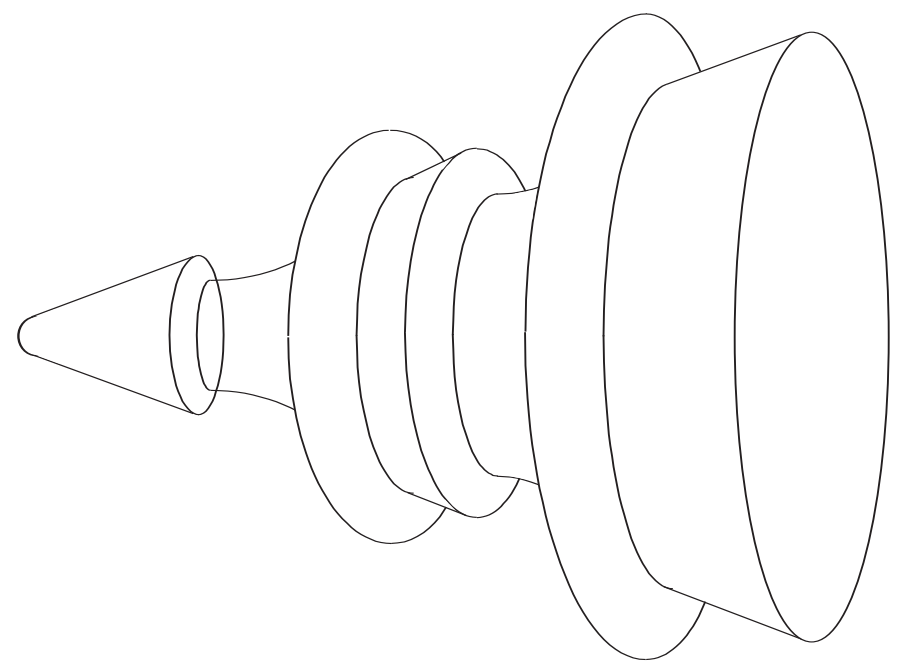

Figure 5 Manifold $M$

Finally, let us estimate the ratio $\frac{\sigma^{\prime \prime}}{\sigma}$. We have, by (52),

$$
\sigma^{\prime \prime}(r)=\frac{\eta_{k-1}-\eta_{k}}{\alpha_{k}^{2}\left(a_{k}-b_{k-1}\right)^{2}} \psi^{\prime \prime}\left(\frac{1}{\alpha_{k}} \frac{r-b_{k-1}}{a_{k}-b_{k-1}}\right) .
$$

Since $\sigma(r) \geq \eta_{k}$, then, by (48), (56), and (54),

$$
\begin{aligned}
\left|\frac{\sigma^{\prime \prime}}{\sigma}\right| & \leq C \frac{\eta_{k-1}-\eta_{k}}{\alpha_{k}^{2}\left(a_{k}-b_{k-1}\right)^{2} \eta_{k}} \\
& \leq C_{1} \frac{\frac{\log b_{k-1}}{b_{k-1}} \log ^{2} b_{k-1}}{b_{k-1}^{2} \frac{\log b_{k}}{b_{k}}} \\
& \leq C_{2} \frac{\log ^{3} b_{k-1}}{b_{k-1}^{3}} \frac{b_{k}}{\log b_{k}} \\
& \leq C_{3} \frac{\log ^{2} b_{k}}{b_{k}^{2-3 / n}} \longrightarrow 0, \quad k \rightarrow \infty .
\end{aligned}
$$

Thus, we see from (48), (57) and (58) that manifold $M$ has bounded curvature and, moreover, is asymptotically flat.

The manifold $M$ is sketched in Fig. 5. Let us note that the shape of $M$ is determined by the function $S(r)$. In our example, this function is oscillating but, nevertheless, $\lim _{r \rightarrow \infty} S(r)=\infty$. Indeed, if $r \in\left(a_{k}, b_{k}\right)$, then, by (18), (49), (22), (46) and (48),

$$
S(r)=\sigma(r) V(r) \geq \eta_{k} V\left(a_{k}\right)=\eta_{k} a_{k}^{n}=c \eta_{k} b_{k}^{n-1} \sim c b_{k}^{n-2} \log b_{k} .
$$


If $r \in\left(b_{k-1}, a_{k}\right)$, then, by (53),

$$
S(r)=\sigma(r) V(r) \geq \eta_{k} V\left(b_{k-1}\right)=2^{-n} \eta_{k} a_{k}^{n} \sim c^{\prime} b_{k}^{n-2} \log b_{k} .
$$

In both cases, we see that $S(r) \rightarrow \infty$ as $k \rightarrow \infty$.

\section{References}

[1] Coulhon T., Grigor'yan A., 'On-diagonal lower bounds for heat kernels on non-compact manifolds and Markov chains', Duke Math. J., 89 (1997) no.1, 133-199.

[2] Gaffney M. P., 'The conservation property of the heat equation on Riemannian manifolds', Comm. Pure Appl. Math., 12 (1959) 1-11.

[3] Grigor'yan A., 'On stochastically complete manifolds', (in Russian) DAN SSSR, 290 (1986) no.3, 534-537. Engl. transl. Soviet Math. Dokl., 34 (1987) no.2, 310-313.

[4] Grigor'yan A., 'Escape rate of Brownian motion on weighted manifolds', Applicable Analysis, 71 (1999) no.1-4, 63-89.

[5] Grigor'yan A., Kelbert M., 'Range of fluctuation of Brownian motion on a complete Riemannian manifold', Ann. Prob., 26 (1998) 78-111.

[6] Itô K., McKean H., Diffusion processes and their sample paths Springer, Berlin, 1965.

[7] Karp L., Li P., 'The heat equation on complete Riemannian manifolds', unpublished manuscript 